\title{
Unmet Needs in the Management of Type 2 Diabetes Mellitus Patients Starting Insulin Therapy with Basal or Premix Insulin in Routine Clinical Practice in Serbia
}

\author{
Teodora M. Beljić Živković (1) • Boris J. Đinđić • Dušica Ž. Šuluburić Toljić • \\ Koviljka T. Milenković Vulović • Dragana L. Udovičić • \\ Dragan Z. Zdravković
}

Received: September 10, 2018 / Published online: November 19, 2018

(c) The Author(s) 2018

\section{ABSTRACT}

Introduction: Type 2 diabetes mellitus (T2DM) is a progressive disease with declining beta cell function, ultimately necessitating insulin therapy. Timely introduction of adequate insulin improves management of diabetes. The aim of this study was to evaluate the unmet needs in the management of T2DM patients recently initiated on insulin therapy in routine clinical practice in Serbia.

Enhanced digital features To view enhanced digital features for this article go to https://doi.org/10.6084/ m9.figshare.7271348.

T. M. Beljić Živković ( $\)$

Division of Endocrinology, Diabetes and Metabolic Disorders, "Zvezdara" University Medical Center, Belgrade University School of Medicine, Belgrade, Serbia

e-mail: dorabeljic@beotel.net

\section{B. J. Đinđić}

Faculty of Medicine, Clinic of Cardiology, Institute of Pathophysiology, Clinical Center Niš, Niš, Serbia

D. Ž. Šuluburić Toljić · K. T. Milenković Vulović General Hospital Čačak, Čačak, Serbia

D. L. Udovičić

Health Center Užice, Užice, Serbia

D. Z. Zdravković

Sanofi, Belgrade, Serbia
Methods: The NEED study was a cross-sectional, observational, multicenter, real-world study conducted in Serbia, involving 26 physicians, endocrinologists, treating individuals with T2DM from 17 secondary health care institutions. Study participants were newly initiated with insulin therapy, being treated with basal or premix insulin \pm oral antidiabetics (OAD) for 6-12 months.

Results: Four hundred one individuals were included in the study between October 2016 and March 2017. The mean age of study patients was $61.8 \pm 9.2$ years with mean BMI $30.0 \pm 5.0 \mathrm{~kg} / \mathrm{m}^{2}$, and duration of diabetes, prior to initiation of insulin therapy, was $8.4 \pm 5.9$ years. A basal insulin regimen was used by $287(71.6 \%)$ and premix insulin by 114 $(28.4 \%)$ subjects. The average daily dose $(39.8 \pm 13.9$ units premix vs. $26.3 \pm 13.5$ units basal), dose $/ \mathrm{kg}(0.47 \pm 0.15$ units $/ \mathrm{kg}$ premix vs. $0.31 \pm 0.17$ units $/ \mathrm{kg}$ basal), and number of injections per day were higher in the premix compared with basal insulin regimen $(p<0.01)$. The percentage of T2DM participants with at least one unmet need was high $(95.8 \%)$. The majority of participants had two or three unmet needs. The most common unmet needs were: HbA1c $>7.0 \%$ (79.3\%), at least one documented symptomatic hypoglycemia $(\leq 3.9$ $\mathrm{mmol} / \mathrm{l})$ event in the previous 3 months $(63.8 \%)$, and two or more doses of insulin per day $(53.1 \%)$. The mean individual HbA1c target was $6.8 \%$ in the NEED study cohort, with only 
$16 \%$ of participants reaching it. Most participants [281 (70.1\%)] experienced symptomatic hypoglycemia.

Conclusions: The NEED study showed that new insulin users of either basal or premix HM insulin have many unmet needs in the first 6-12 months of treatment. This confirms that in reallife settings novel insulins should be considered in the management of T2DM to reduce the number of symptomatic hypoglycemic events and reach a better HbA1c level.

Funding: Sanofi, Serbia.

Keywords: Basal insulin; Diabetes; Premix insulin; Type 2 diabetes; Unmet needs

\section{INTRODUCTION}

Type 2 diabetes mellitus (T2DM) is a progressive disease with declining beta cell function, ultimately necessitating introduction of insulin therapy. Despite well-documented benefits of timely glycemic control and consensus guidelines encouraging early use of insulin, considerable clinical inertia exists with respect to initiating appropriate insulin therapy in people with T2DM [1]. Furthermore, a significant proportion of patients already treated with basal insulin is failing to achieve glycemic targets in the real world [2].

Insulin therapy in Serbia is fully reimbursed by the government. However, there are some limitations related to the choice of insulin therapy. Full reimbursement for the introduction of insulin therapy is for human (HM) insulins only. After failure to achieve good glycemic control with $\mathrm{HM}$ for a period of 6 months, with documented hypoglycemia and HbA1c levels higher than 7\%, insulin analogs can be introduced and fully reimbursed.

International Diabetes Federation (IDF) clinical practice recommendations define management of type 2 diabetes mellitus (T2DM) in primary practice [3]. In Serbia, HM insulin therapy is usually initiated in the inpatient setting at the secondary levels or at the outpatient clinic at the tertiary level.

The NEED study was conducted to evaluate the unmet needs in the management of T2DM patients treated with basal or premix insulins in routine clinical practice in Serbia.

\section{METHODS}

\section{Study Design and Population}

The NEED study was a cross-sectional, observational, multicenter study involving 26 physicians, endocrinologists, treating individuals with T2DM from 17 secondary health care institutions in Serbia. The participating sites were equally geographically distributed across the country to ensure a good representation of the insulin management of T2DM patients. Each physician had to include up to 20 consecutive subjects, fulfilling the inclusion/exclusion criteria. Patients were enrolled consecutively from the cohort seen by the participating physicians in their daily clinical practice. According to the non-interventional study design, all treatments and procedures were performed at the physicians' discretion and did not interfere with everyday clinical practice.

Participants were adult patients with T2DM, 18 years of age or older, on basal or premix HM insulin as initial regimen for 6-12 months, with or without oral antidiabetic drugs (OADs). They had to provide HbA1c measurements, performed in the last 3 months, and a patient diary with information on insulin doses and recordings of hypoglycemic events from the last 3 months. Potential T1DM patients $(<40$ years of age who started insulin within a year after diabetes diagnosis), those on systemic corticosteroids, pregnant or breastfeeding women, and those already taking part in other studies were excluded from the study. The participants signed an informed consent form prior to the conduct of any study-related procedures.

According to the cross-sectional study design, data collection was done at only one visit, and some data were collected retrospectively from the electronic records. The anamnestic and clinical data were collected, including body weight and height, HbA1c level (not older than 3 months), diabetes history and complications, other comorbidities, insulin 
treatment and concomitant antidiabetic medications, individualized $\mathrm{HbA1c}$ target defined by the physicians, and history of severe, symptomatic, and verified symptomatic hypoglycemia (within the last 3 months prior to study entry) documented in the patient diary. The participants were asked to fill in the fear of hypoglycemia questionnaire. Participating physicians collected the data and information requested by protocol in an electronic case report form (e-CRF).

The primary objective of this study was to estimate the percentage of participants with unmet needs (at least 1 of the 5 defined needs) in T2DM patients started on insulin (basal or premix3) with/without OAD for the first 612 months. Unmet needs were defined as: (1) uncontrolled glycemia-participants not reaching HbA1c $\leq 7 \%$; (2) severe hypoglycemia (requiring assistance of another person to actively administer carbohydrate, glucagon, or other resuscitative actions) in the previous 3 months; (3) documented symptomatic hypoglycemia $(\leq 3.9 \mathrm{mmol} / \mathrm{l})$ in the previous 3 months; (4) two or more doses of insulin per day; (5) weight gain $\geq 3 \%$ of body weight since insulin initiation.

The secondary objectives included the proportion of patients with each unmet medical need and the combination of two or more unmet needs, proportion of patients on target HbA1c $<7 \%$, HbA1c $<8 \%$, and individualized HbA1c, proportion of patients on target HbA1c $<7.0 \%$ without symptomatic hypoglycemia within 3 months and weight gain $\geq 3 \%$ since insulin initiation, proportion of patients on target $\mathrm{HbA} 1 \mathrm{c}<7 \%$ without severe hypoglycemia within 3 months prior to study entry, incidence of hypoglycemic events within 3 months of study entry, and fear of hypoglycemia, assessed by Hypoglycemia Fear Survey II (HFS II). Signed informed consent was obtained from all participants. The study was approved by the local ethics committees and conducted in accordance with Good Clinical Practice and the 1964 Declaration of Helsinki and its later amendments or comparable ethical standards.

\section{Statistical Analysis}

All variables are presented as continuous (scale) or categorical (nominal and ordinal) data. Continuous data are presented as mean values with standard deviations or as medians with interquartile ranges (for skewed data), and categorical data are presented by absolute numbers with percentages in descriptive statistics. Depending of the type and number of examined parameters, chi-square, Mantel-Haenszel, and Student's $t$ tests for independent or paired samples as wll as Mann-Whitney test (nonGaussian distribution), Wilcoxon test, ANOVA, and post hoc analyses were used. In all tests, the alpha level at 0.05 ( $p$ value $<0.05$ ) was considered to be statistically significant. Levene's test was used to assume equality of variances. Normal distribution of each variable was for checking with the Kolmogorov-Smirnoff test. Missing data were presented in descriptive analysis with valid percent calculated. In other analysis, they were handled as missing data and excluded from analysis.

The estimated number of patients with diabetes in Serbia is 858,900 [4]. Assuming that $30 \%$ of T2DM patients require insulin therapy, the prevalence of these patients represents about 257,000 . Supposing $50 \%$ frequency of unmet needs, a margin of error of $\pm 5.1 \%$ (44.9\%; $55.1 \%$ ), and confidence level of $95 \%$ (alpha: 0.05), the minimum sample size is 360 participants. This interval has a length of $10.2 \%$, indicating a precise estimate. Considering the possibility of $10 \%$ missing data, the final sample size is corrected to 400 participants.

\section{RESULTS}

\section{Characteristics of Participants}

In the NEED study in Serbia, a total of 401 individuals with T2DM, newly initiated to insulin therapy, were included between October 2016 and March 2017. There were 203 (50.6\%) male and 198 (49.4\%) female participants. The mean age was $61.8 \pm 9.2$ years, with predominance of participants from 60 to 70 years. The males included in the study were significantly 
younger $(60.94 \pm 9.3$ vs. $62.9 \pm 9.0$ years $)$ than females $(p<0.05)$. The mean body mass index (BMI) was $30.0 \pm 5.0 \mathrm{~kg} / \mathrm{m}^{2}$ and was significantly higher in females (females $30.5 \pm 5.0 \mathrm{~kg}$ / $\mathrm{m}^{2}$ vs. males $\left.29.5 \pm 5.0 \mathrm{~kg} / \mathrm{m}^{2} ; p<0.05\right)$.

The mean duration of T2DM was $8.4 \pm 5.9$ years (median: 7.6 years), and mean duration of oral antidiabetic therapy was $8.0 \pm 5.6$ years (median: 7.4 years). Neuropathy was the most common microvascular complication of diabetes (55.5\%), being mostly peripheral sensitive neuropathy (98.6\%). Retinopathy was present in $21.5 \%$ of participants, and it led to blindness in 5.8\%. Among the participants with nephropathy (13.0\%), end-stage renal failure was present in $1.9 \%$, advanced kidney disease in $23.0 \%$, proteinuria in $21.3 \%$, and albuminuria in $53.8 \%$. Comorbidity was found in 286 (71.3\%), dyslipidemia and arterial hypertension being most frequent (Table 1).

\section{Insulin and Concomitant Antidiabetic Therapy}

The average duration of insulin therapy was $8.99 \pm 2.01$ months, median 9.03 months. A human intermediate-acting basal NPH insulin regimen was used by 287 (71.6\%) and HM premix 30/70 insulin by $114(28.4 \%)$ subjects. The majority of basal insulin users [240 (84\%)] took human intermediate-acting basal insulin (NPH), and $47(16 \%)$ used basal analogs (determir or glargine). The average daily doses and number of injections per day are shown in Table 2 . The average daily dose, dose $/ \mathrm{kg}$, and number of injections per day were higher in the premix than basal insulin regimen $(p<0.01)$.

In total, $349(99.5 \%)$ had concomitant antidiabetic therapy. The proportion of different drug classes per insulin regimen after insulin initiation is shown in Table 2. Metformin and sulfonylureas were the predominant glucose-lowering medications taken concomitantly with insulin. The sulfonylureas were significantly more commonly prescribed in participants with basal insulin than premix insulin.
Table 1 Prevalence of comorbidities

\begin{tabular}{lcl}
\hline Comorbidity & \multicolumn{1}{l}{$\boldsymbol{n}$} & \multicolumn{1}{l}{$\%$} \\
\hline Hypertension & 340 & 84.8 \\
Dyslipidemia & 306 & 76.3 \\
Coronary heart disease & 97 & 24.3 \\
Acute myocardial infarction & 27 & 6.8 \\
Myocardial revascularization & 23 & 5.8 \\
Heart failure & 38 & 9.5 \\
Atrial fibrillation & 19 & 4.8 \\
Stroke & 19 & 4.8 \\
Peripheral vascular disease & 44 & 11 \\
Peripheral revascularization & 8 & 2 \\
Foot ulcer & 9 & 2.3 \\
Lower limb amputation & 5 & 1.3 \\
Fatty liver disease & 97 & 24.3 \\
Chronic obstructive pulmonary disease & 29 & 7.3 \\
Obstructive sleep apnea & 11 & 2.8 \\
Hip fracture/osteoporosis & 13 & 3.3 \\
Malignant disease & 11 & 2.8 \\
Depression & 38 & 9.5 \\
Severe dementia & 2 & 0.5 \\
\hline
\end{tabular}

\section{Presence, Type, and Number of Defined Unmet Needs}

The percentage of T2DM participants with at least one of the five defined unmet needs was $95.8 \%$, independent of type of insulin regimen and type of basal insulin used (Table 3). Proportions of patients with documented symptomatic hypoglycemia according to insulin therapy did not show significant differences between insulin analogs [29 (61.7\%)] and human intermediate [162 (67.5\%)].

The most common unmet needs were: HbA1c $>7.0 \%$ (79.3\%), at least one documented symptomatic hypoglycemia $(\leq 3.9 \mathrm{mmol} / \mathrm{l})$ event in the previous 3 months (63.8\%), and two or more doses of insulin per day (53.1\%). The 
Table 2 Characteristics of insulin and concomitant antidiabetic therapy

\begin{tabular}{llll}
\hline & Basal insulin & Premix insulin & Total \\
\hline$N / \%$ & $287 / 71.6$ & $114 / 28.4$ & $401 / 100.0$ \\
Average HbAlc (\%) & $7.92 \pm 1.3$ & $7.94 \pm 1.2$ & $7.92 \pm 1.3$ \\
Dose (U) & $26.3 \pm 13.5$ & $39.8 \pm 13.9^{*}$ & $30.2 \pm 14.9$ \\
Dose (U/kg) & $0.31 \pm 0.17$ & $0.47 \pm 0.15^{*}$ & $0.35 \pm 0.18$ \\
Once daily $(n / \%)$ & $182 / 63.4$ & $6 / 5.3$ & $188 / 46.9$ \\
Twice daily $(n / \%)$ & $83 / 28.9$ & $100 / 87.7^{\dagger}$ & $183 / 45.6$ \\
Three times daily $(n / \%)$ & $22 / 7.7$ & $8 / 7.0$ & $30 / 7.5$ \\
Metformin & $251 / 87.4$ & $105 / 92.1$ & $356 / 88.8$ \\
Sulfonylureas (SU) & $76 / 26.5$ & $10 / 8.8^{\ddagger}$ & $86 / 21.4$ \\
Metiglinidis (glinides) & $6 / 2.0$ & $0 / 0.0$ & $6 / 1.5$ \\
Thiazolidinediones & $2 / 0.6$ & $0 / 0.0$ & $2 / 0.5$ \\
GLP1 receptor agonists & $2 / 0.6$ & $2 / 1.8$ & $4 / 1.0$ \\
DPP-IV inhibitors & $8 / 2.7$ & $0 / 0.0$ & $8 / 2.0$ \\
SGLT-2 inhibitors & $6 / 2.1$ & $0 / 0.0$ & $6 / 1.5$ \\
\hline
\end{tabular}

Data are presented as $\mathrm{X} \pm \mathrm{SD}$ or $n / \%$, Student's $t$ test ${ }^{*} p<0.01$ vs. basal; chi-square test ${ }^{\dagger} p<0.01$ vs. basal; Pearson chisquare test ${ }^{*} p<0.05$ vs. basal

Table 3 Proportion of T2DM patients with at least one of the defined unmet needs

\begin{tabular}{lllll}
\hline \multicolumn{4}{l}{ Type of current insulin treatment } & \\
\cline { 2 - 5 } & Human intermediate & Long-acting analog & Premix insulin & Total \\
\hline No unmet need & $14 / 5.8$ & $0 / 0.0$ & $3 / 2.6$ & $17 / 4.2$ \\
With unmet need & $226 / 94.2$ & $47 / 100.0$ & $111 / 97.4$ & $384 / 95.8$ \\
Total & $240 / 100.0$ & $47 / 100.0$ & $114 / 100.0$ & $401 / 100.0$ \\
\hline
\end{tabular}

Non-significant between different types of insulin used

proportion of participants with documented symptomatic hypoglycemia was significantly higher $(p<0.05)$, and the proportion of participants with $\geq$ two doses of insulin daily was significantly lower in the group with the basal insulin regimen compared with the premix insulin $(p<0.01)$ (Table 4$)$.

The proprotion of patients with documented symptomatic hypoglycemia using SU was higher than in patients not using SU (64/86 vs.
192/315, $p<0.05)$. After excluding patients with SU, the proportion of patients with documented symptomatic hypoglycemia was similar in both insulin regimens (135/211 in the basal insulin and $57 / 104$ patients in the premix group). The majority of participants had two (33.9\%) and three unmet needs (32.7\%). HbA1c $>7 \%$ with documented symptomatic hypoglycemia was the most common dual combination followed by HbA1c $>7 \%$ with $\geq 2$ 
Table 4 Presence, type, and number of defined unmet needs per insulin regimen

\begin{tabular}{llll}
\hline & Basal insulin & Premix insulin & Total \\
\hline No unmet needs & $14 / 4.9$ & $3 / 2.6$ & $17 / 4.2$ \\
With unmet needs & $273 / 95.1$ & $111 / 97.4$ & $384 / 95.8$ \\
HbA1c $>$ 7\% & $225 / 78.4$ & $93 / 81.6$ & $318 / 79.3$ \\
Severe hypoglycemia 3 months prior to study entry & $44 / 15.3$ & $15 / 13.2$ & $59 / 14.7$ \\
Documented symptomatic hypoglycemic event & $191 / 66.5^{*}$ & $65 / 57.0$ & $256 / 63.8$ \\
Documented symptomatic hypoglycemic event-patients without SU & $135 / 63.9$ & $57 / 54.8$ & $192 / 61.0$ \\
$\geq 2$ Doses daily & $105 / 36.5^{\dagger}$ & $108 / 94.7$ & $213 / 53.1$ \\
Weight gain $\geq 3 \%$ & $76 / 26.5$ & $27 / 23.7$ & $103 / 25.7$ \\
One medical unmet need & $58 / 20.2$ & $5 / 4.4$ & $63 / 15.7$ \\
Two medical unmet needs & $97 / 33.8$ & $39 / 34.2$ & $136 / 33.9$ \\
Three medical unmet needs & $85 / 2.6$ & $4 / 40.4$ & $131 / 32.7$ \\
Four medical unmet needs & $31 / 10.8$ & $18 / 15.8$ & $49 / 12.2$ \\
Five medical unmet needs & $2 / 0.7$ & $3 / 2.6$ & $5 / 1.2$ \\
\hline
\end{tabular}

Data are presented as $n / \%$, chi-square test; ${ }^{*} p<0.05 ;{ }^{\dagger} p<0.01$ vs. premix insulin

doses daily. HbA1c $>7 \%$ with documented symptomatic hypoglycemia and $\geq 2$ doses daily was the most common triple combination. The participants on the basal insulin regimen most commonly had two unmet needs (33.8\%), while participants with premix insulin mostly had three unmet needs (40.4\%).

\section{Proportion of Patients on Target Glycemic Control}

The mean value of the last measured HbA1c (within 3 months before study entry) was $7.9 \pm 1.3 \%$. The mean individualized target HbA1c was $6.8 \pm 0.4 \%$. The most common individual HbA1c targets were under $7.0 \%$ and $7-7.5 \%$, respectively, in $43.1 \%$ and $47.4 \%$. Only $6.5 \%$ of patients had target $\mathrm{HbA} 1 \mathrm{c} 7.5-8 \%$, and $3 \%$ of patients had target HbA1c $>8 \%$. The major reasons underlying physicians' decisions on setting less stringent individualized HbA1c targets were participant age (70\%), patient acceptance of treatment (54\%), and comorbidities (53\%). Only $16.2 \%$ of study participants reached the individualized target HbA1c level set by their physician.

In total, $17.2 \%$ of participants had $\mathrm{HbA} 1 \mathrm{c}<7.0 \%$ and $59.6 \%$ HbA1c $<8.0 \%$; $82.3 \%$ of participants were high risk (participants $\geq 65$ years old or with evidence of any of the comorbidities/characteristics such as chronic complications and cardiovascular comorbidities, prior severe hypoglycemic events, and professional driving). Among highrisk participants, significantly fewer participants had HbA1c $<7 \%(14.8 \%)$ compared with lowrisk participants (28.2\%). There was no significant difference in the proportion of participants having HbA1c $<8 \%$ according to the level of risk.

Only $24(6 \%)$ participants had the target HbA1c level $(<7.0 \%)$ without symptomatic hypoglycemia in the previous 3 months and no weight gain $\geq 3 \%$. The analysis showed no statistical difference between the group on the basal insulin regimen (9 subjects, $6.6 \%$ ) and the group on premix insulin (5 subjects, 4.4\%). Sixty-three (15.7\%) participants achieved the target HbA1c $(<7.0 \%)$ without severe 
hypoglycemia within 3 months prior study entry, with no difference between basal insulin regimens.

\section{Incidence of Hypoglycemic Events and Fear of Hypoglycemia, Assessed by HFS}

In the last 3 months, prior to study entry, most participants [281 (70.1\%)] experienced symptomatic hypoglycemia. Documented symptomatic events were registered in $256(63.8 \%)$ and severe hypoglycemic events in 59 (14.7\%) participants. There were 12.83 symptomatic hypoglycemic events, 9.01 documented symptomatic events, and 0.77 severe hypoglycemic events per patient-year. The average HFS total score was $67.2 \pm 27.3$.

\section{DISCUSSION}

This observational study gives an overview of the unmet needs in the insulin management of newly initiated T2DM patients in everyday clinical practice in Serbia. The NEED study has shown that there were no major differences in baseline characteristics of subjects introduced to insulin in Serbia compared with other observational studies performed in Europe. As expected, patients with T2D were older (mean age $61.8 \pm 9.2$ years) with males significantly younger than females. Mean BMI was $30.0 \pm 5.0 \mathrm{~kg} / \mathrm{m}^{2}$ and significantly higher in females. Similar data were obtained from another observational study in different parts of Europe; however, the duration of T2DM in our study was shorter than in data in European countries [5]. Duration of diabetes when starting insulin therapy seems to be around 10 years worldwide [6], but duration of uncontrolled hyperglycemia before insulin initiation is still questionable. Hence, the prevalence of vascular complications is variable between studies. The ADHOC study in France [7] showed a relatively high prevalence of diabetic complications (neuropathy: 25\%; fundus' abnormalities: 27\%, angina pectoris: $13 \%$; myocardial infarction: 10\%; stroke: 6\%; lower-limb arteritis: $15 \%$ ) and risk factors, particularly hypertension $(77.5 \%)$ and dyslipidemia (71\%). The NEED study in Serbia registered more microvascular complications (neuropathy in $55.5 \%$ of patients) than in the French cohort, which could indicate poor metabolic control in participants. Hypertension and dyslipidemia were also more prevalent than in the French study.

Normalizing blood glucose level is the important factor for preventing vascular complications. When the HbA1c target is not reached while on OADs, insulin therapy is introduced (since the local reimbursement policy does not allow GLP-1RA initiation). Factors influencing the initial choice of insulin therapy were evaluated in a large international non-interventional study [8]. In this study, insulin was started as basal insulin alone in $51.6 \%$ $(15.7 \pm 11.0 \mathrm{U} /$ day $)$, basal \pm mealtime insulin $14.6 \%(23.3 \pm 17.0 \mathrm{U} /$ day $)$, or premix alone in $23.1 \%$ (20.9 $\pm 14.1 \mathrm{U} /$ day). Physicians preferred basal insulin when HbA1c was less elevated and with prior secretagogue therapy. A similar pattern in choosing initial insulin regimens, but with higher insulin doses, was observed in the NEED study. The average daily dose and number of injections per day were significantly higher in the premix than basal insulin regimen in the NEED study, similar to the mentioned study. Contrarily, the basal insulin regimen was associated with a higher starting HbA1c level compared with premix insulin. In our study, $88.8 \%$ of the patients were taking metformin together with insulin and only $21.4 \%$ were using sulfonylureas. Similar to other observational studies, the sulfonylureas were more commonly prescribed in patients on basal insulin regimens and less often in patients on premix insulin [5].

Almost all participants (95.8\%) had at least one defined unmet need, independent of type of insulin used. The most common unmet need was HbA1c $>7.0 \%$, found in almost $80 \%$ of participants. Documented symptomatic hypoglycemia was present in two thirds and use of $\geq 2$ doses of insulin per day in half of the participants. Around $15 \%$ of patients reported severe hypoglycemia. The majority of participants had two (33.9\%) or three unmet needs $(32.7 \%)$. The participants on basal insulin regimens most commonly had two unmet needs 
(33.8\%) while participants with premix insulin most commonly had three unmet needs (40.4\%).

The mean individual HbA1c target was $6.8 \%$ in the NEED study cohort, and only $16 \%$ of participants reached their individual HbA1c target, regardless of initiated insulin regimen. In total, $17.2 \%$ participants had HbA1c under $7.0 \%$ and $59.6 \%$ had HbA1c under $8.0 \%$. Symptomatic hypoglycemia was experienced by $70 \%$ of our participants. There were 0.77 severe hypoglycemic events/patient/year, more than in the Fremantle diabetes study [8]. This can be explained by the use of HM insulins $(84 \%$ of basal regime users were on HM insulin, and this group represents $71.6 \%$ of all NEED study participants) and great prevalence of neuropathy, which could blunt hypoglycmemia symptoms [9]. Irrespective of intrinsic differences between data sources, $24-54 \%$ of patients with T2DM globally had residual hyperglycemia with HbA1c not at target, despite achieving FPG control [10]. A similar study design showed documented hypoglycemia in $28-39 \%$ of patients with premix insulin and $35-41 \%$ with basal insulins [11], almost half of the prevalence registered in the NEED cohort. This difference indicates a gap that might be explained by different participant educational levels and adherence to the insulin titration recommendation given by physicians. Other authors found that contributing factors to this effective treatment gap likely include lack of health education and poor medicine adherence by some patients [12]. In addition, it is possible that the type of insulin being used (human intermediate-acting or human premix), as per current reimbursement limitations in Serbia, contributes to these findings of a high prevalence of participants with present unmet needs. Use of sulfonylureas may have have contributed to the hypoglycemic events in very few subjects as the dose of these drugs is always reduced from maximal to optimal on starting insulin.

A similar proportion of patients had HbA1c $<7.0 \%$, no symptomatic hypoglycemia, and no weight gain $\geq 3 \%$, including the proportion of patients with $\mathrm{HbA} 1 \mathrm{c}<7.0 \%$ and without severe hypoglycemia, according to type of insulin therapy. Although no significant difference in any hypoglyemic prevalence and composite targets (including weight gain $\geq 3 \%$ ) was found between the groups in the NEED cohort, the meta-analysis of other studies confirmed that HM + OAD has a better safety profile than premix + OAD [13]. The literature data confirm that weight gain occurs early with the initiation of insulin therapy and has been reported to vary from 0.3 to $6.4 \mathrm{~kg}$. Therapy with basal insulin induces less weight gain than premix insulin [14].

A significant proportion of patients experienced symptomatic and documented symptomatic hypoglycemic events with poor HFS total scores. This indicates a need for improved titration algorithms, better insulin therapy education of patients, and development of newer insulin formulations aimed at reducing the proportion of patients experiencing hypoglycemia.

The study may have been limited by several factors: the observational design of the study with a relatively short time frame and the retrospective nature of hypoglycemia collection, not using standardized patient diaries and HbA1c. Perhaps the main limitation was that the results are mainly derived from HM insulin treatment and sulfonylurea derivates, mostly outdated in other European counties, because of current local reimbursement restrictions. Due to the reimbursement criteria in Serbia, we included a relatively small number of patients with basal analogs in the study. This could be a limiting factor in the statistical analysis as well as an explanation for the high hypoglycemic event incidence and relatively small number of patients reaching the target HbA1c.

However, our results provide a useful perspective on the management of early insulin users by diabetologists in Serbia and the need for novel therapeutic agents in achieving better glycemic control with fewer adverse events.

\section{CONCLUSION}

The NEED study in Serbia has shown that new insulin users of either NPH basal or HM premix insulin are mainly older subjects, with long duration of diabetes and presence of chronic 
complications and comorbidities. The majority of patients did not reach HbA1c targets 6-12 months after insulin initiation, experienced symptomatic hypoglycemia, and presented with at least one unmet medical need regardless of insulin regimen. This confirms that in reallife settings, novel insulins should be considered in the management of T2DM to reduce symptomatic hypoglycemic events and to reach a better HbA1c level.

\section{ACKNOWLEDGEMENTS}

We thank the study participants.

Funding. The NEED study and article processing charges for this article were fully sponsored by Sanofi, Serbia. All authors had full access to all of the data in this study and take complete responsibility for the integrity of the data and accuracy of the data analysis.

Authorship. All named authors meet the International Committee of Medical Journal Editors (ICMJE) criteria for authorship for this manuscript, take responsibility for the integrity of the work as a whole, and have given final approval to the version to be published.

Authorship Contributions. Teodora M. Beljić Živković was the main and corresponding author involved in writing, reviewing, and editing the manuscript and had final responsibility for approving the published version of the manuscript. Boris J. Djindjić was involved in creating the study design, performed the statistical analyses of the data, and was involved in writing, reviewing, and editing the manuscript with approval of the final version. Dušica Ž. Šuluburić Toljić, Koviljka T. Milenković Vulović, Dragana L. Udovičić, and Dragan Z. Zdravkovic were involved in writing, reviewing, and editing the manuscript and providing the final approvals.

Disclosures. Teodora M. Beljić Živković declares associations (Lecturer, Member of the Advisory Board, Clinical Trial Investigator) with the following companies: AstraZeneca, Boehringer-Ingelheim, Berlin-Chemie, Eli Lilly, Merck Sharpe \& Dohme, Novartis, NovoNordisk, and Sanofi-Aventis. Boris J. Djindjić declares associations (Lecturer, Member of the Advisory Board, Clinical Trial Investigator) with the following companies: Boehringer-Ingelheim and Sanofi-Aventis. Dušica Ž. Šuluburić Toljić declares associations (Lecturer, Member of the Advisory Board, Clinical Trial Investigator) with the following companies: Boehringer-Ingelheim, Berlin-Chemie, NovoNordisk, and SanofiAventis. Koviljka T. Milenković Vulović declares associations (Lecturer, Member of the Advisory Board, Clinical Trial Investigator) with the following companies: Boehringer-Ingelheim, Berlin-Chemie, NovoNordisk, and Sanofi-Aventis. Dragana L. Udovičić declares associations (Lecturer, Member of the Advisory Board, Clinical Trial Investigator) with the following companies: AstraZeneca, Boehringer-Ingelheim, Berlin-Chemie, Eli Lilly, Merck Sharpe \& Dohme, Novartis, NovoNordisk, and Sanofi-Aventis. Dragan Z. Zdravković is a Sanofi employee.

Compliance with Ethics Guidelines. All procedures followed were in accordance with the ethical standards of the responsible committee on human experimentation (institutional and national) and with the Helsinki Declaration of 1964, as revised in 2013. Informed consent was obtained from all subjects prior to inclusion in the study.

Data Availability. The data sets during and/ or analyzed during the current study are available from the corresponding author on reasonable request.

Open Access. This article is distributed under the terms of the Creative Commons Attribution-NonCommercial 4.0 International License (http://creativecommons.org/licenses/ by-nc/4.0/), which permits any noncommercial use, distribution, and reproduction in any medium, provided you give appropriate credit to the original author(s) and the source, provide a link to the Creative Commons license, and indicate if changes were made. 


\section{REFERENCES}

1. Khunti K, et al. Study of Once Daily Levemir $\left(\mathrm{SOLVE}^{\mathrm{TM}}\right)$ : insights into the timing of insulin initiation in people with poorly controlled type 2 diabetes in routine clinical practice. Diabetes Obes Metab. 2012;14(7):654-61. https://doi.org/10.1111/ j.1463-1326.2012.01602.x.

2. Dalal MR, Grabner M, Bonine N, Stephenson JJ, DiGenio A, Bieszk N. Are patients on basal insulin attaining glycemic targets? Characteristics and goal achievement of patients with type 2 diabetes mellitus treated with basal insulin and physician-perceived barriers to achieving glycemic targets. Diabetes Res Clin Pract. 2016;121:17-26.

3. IDF Clinical practice recommendations for managing type 2 diabetes in primary care. International Diabetes Federation, 2017. https://www.idf.org/elibrary/guidelines/128-idf-clinical-practice-recomm endations-for-managing-type-2-diabetes-in-primarycare.html.

4. Institute of Public Health of Serbia "Dr Milan Jovanovic Batut". Report No 9. Incidence and mortality of diabetes in Serbia, 2014. http://www. batut.org.rs/download/publikacije/2014IzvestajDija betes.pdf.

5. Blonde L, Marre M, Vincent M, Brette S, Pilorget V, Danchin N, Vespasiani G, Home P. Insulin regimens and glycemic control in different parts of Europe over 4 years after starting insulin in people with type 2 diabetes: data from the CREDIT noninterventional study. Diabetes Res Clin Pract. 2017;133:150-8.

6. Home PD, Dain MP, Freemantle N, Kawamori R, Pfohl M, Brette S. Four-year evolution of insulin regimens, glycaemic control, hypoglycaemia and body weight after starting insulin therapy in type 2 diabetes across three continents. Diabetes Res Clin Pract. 2015;108:350-9.

7. Penfornis A, San-Galli F, Cimino L, Huet D. Current insulin therapy in patients with type 2 diabetes: results of the ADHOC survey in France. Diabetes Metab. 2011;37:440-5.

8. Freemantle N, Balkau B, Danchin N, Wang E, Marre M, Vespasiani G, Kawamori R, Home PD. Factors influencing initial choice of insulin therapy in a large international non-interventional study of people with type 2 diabetes. Diabetes Obes Metab. 2012;14:901-9.

9. Akram K, Pedersen-Bjergaard U, Carstensen B, Borch-Johnsen K, Thorsteinsson B. Frequency and risk factors of severe hypoglycaemia in insulintreated type 2 diabetes: a cross-sectional survey. Diabet Med. 2006;23:750-6.

10. Raccah D, Chou E, Colagiuri S, Gaàl Z, Lavalle F, Mkrtumyan A, et al. A global study of the unmet need for glycemic control and predictor factors among patients with type 2 diabetes mellitus who have achieved optimal fasting plasma glucose control on basal insulin. Diabetes Metab Res Rev. 2017;33(3):e2858.

11. Massi Benedetti M, Humburg E, Dressler A, Ziemen M. A one-year, randomised, multicentre trial comparing insulin glargine with NPH insulin in combination with oral agents in patients with type 2 diabetes. Horm Metab Res. 2003;35(3):189-96.

12. Stokes A, Berry KM, Mchiza Z, Parker WA, Labadarios D, Chola L. Prevalence and unmet need for diabetes care across the care continuum in a national sample of South African adults: evidence from the SANHANES-1, 2011-2012. PLoS One. 2017;12(10):e0184264.

13. Rys P, Wojciechowski P, Rogoz-Sitek A, Niesyczyński G, Lis J, Syta A, Malecki MT. Systematic review and meta-analysis of randomized clinical trials comparing efficacy and safety outcomes of insulin glargine with NPH insulin, premixed insulin preparations or with insulin detemir in type 2 diabetes mellitus. Acta Diabetol. 2015;52:649-62.

14. Lau A, Tang T, Halapy H, Thorpe K, Yu C. Initiating insulin in patients with type 2 diabetes. CMAJ. 2012;184(7):767-76. 